\title{
Antibiotic-Loaded Resorbable Bone-Graft Substitute: A New Treatment for Osteomyelitis/Osteitis
}

\author{
Bernd Gächter ${ }^{1}$, Jennifer Frieda Angehrn ${ }^{2}$, Stephane Schlunke ${ }^{3}$, Sebastian Probst ${ }^{4}$ and Paul Biegger ${ }^{1}$ \\ 1. Department of Surgery, Ospedale Regionale di Locarno, Locarno 6600, Switzerland \\ 2. Department of Obstetrics and Gynecology, Ospedale Regionale di Locarno, Locarno 6600, Switzerland \\ 3. Department of Surgery, Clinica Luganese Moncucco, Lugano 6900, Switzerland \\ 4. University of Applied Sciences Western Switzerland, Geneva 1206, Switzerland
}

\begin{abstract}
Patients with osteomyelitis require lengthy antibiotic treatment, often only to see the inflammation flare up once antibiotics are suspended. Unfortunately, patients often discontinue the antibiotic treatment due to collateral effects. Patients with osteitis are often polymorbid patients with other severe diseases such as diabetes mellitus and polyneuropathy, arteriopathy or polyarthritis with immunosuppression. The eight patients included in the study presented nine bones with osteomyelitis (macroscopically, bacteriologically, histologically or radiologically). The diseased part of the bone was resected, a locally radical debridement was done and a biopsy for bacteriology and histology were taken. The residual bone was then drilled out and filled with antibiotic-loaded (gentamicin) resorbable bone-graft substitute under radiologic imaging control. In total, seven patients are currently without recurrent osteomyelitis with a mean follow-up of 5.77 months (2 11 months). The Kaplan Meier curve shows $80 \%$ survival rate without recurrent osteomyelitis at 11 months. Only one patient suffering from Morbus Buerger had a relapse osteomyelitis after cutting off severing his foot while swimming in the sea. Antibiotic-loaded resorbable bone-graft substitute is easy to use, has in our hands few complications and low recurrence rate.
\end{abstract}

Key words: Osteomyelitis, bioabsorbable antibiotic carrier, bone-graft substitute.

\section{Introduction}

The Locarno Wound Care Center is located in a peripheral area with a population of 120,000 inhabitants. But we also have many patients outside the catchment area (an area with a population of 350,000 inhabitants). Every month one additional patient with a chronic wound including clinical and radiological signs of osteomyelitis o osteitis is referred to the Wound Care Center.

Osteomyelitis or osteitis is an infection and inflammation of the bone marrow or bone $[1,2]$. Because not only the bone marrow but all parts of the bone are affected by the inflammatory process in most cases, the term osteitis is increasingly used instead of osteomyelitis. Bone and joint infections are painful for patients and frustrating for both them and their doctors

Corresponding author: Bernd Gächter, M.D, research fields: wound care and surgery.
[3]. The key to successful management is early diagnosis, including bone sampling for microbiological and pathological examination to allow targeted and long-lasting antimicrobial therapy [3].

Our patients with osteomyelitis require lengthy antibiotic treatment, often only to see the inflammation flare up once antibiotics are suspended. Unfortunately, patients often discontinue the antibiotic treatment due to standing effects. In certain patient populations, osteomyelitis is a significant clinical problem [4]. Patients with osteitis are often polymorphic patients with other severe diseases such as diabetes mellitus and polyneuropathy, arteriopathy or polyarthritis with immunosuppression. Or patients with osteomyelitis are living together with the margins of our society. Drugs and alcohol are highly present and compliance low. Ideal would be a simple therapy that succeeds even in these situations. 
The diagnosis of osteomyelitis is a challenge [5]. An ulcer area larger than $2 \mathrm{~cm}^{2}$, a positive probe-to-bone test result, an erythrocyte sedimentation rate of more than 70 $\mathrm{mm} / \mathrm{h}$, and an abnormal plain radiograph result are helpful in diagnosing the presence of osteomyelitis [5]. Probing for bone should be included in the initial assessment of all patients [6]. Osteomyelitis frequently requires more than one imaging technique for an accurate diagnosis [7]. Conventional radiography still remains the first imaging modality [7]. MRI and nuclear medicine are the most sensitive and specific methods for the detection of osteomyelitis [7]. Radiographs and bone cultures are the mainstays of diagnosis [8]. Osteomyelitis is traditionally staged by the Waldvogel and the Cierny-Mader classification system [9]. The Waldvogel classification represents an etiologic system [9]. The Cierny-Mader classification is based on the anatomy of bone infection and the physiology of the host $[8,9]$.

Generally, a multidisciplinary approach is required for success, involving expertise in orthopedic surgery, infectious diseases, and plastic surgery, as well as vascular surgery, particularly for complex cases with soft-tissue loss [3]. The treatment goal is achieving bone consolidation and avoiding development of chronic osteomyelitis. Successful treatment requires adequate surgical procedures combined with $6 \sim 12$ weeks of antimicrobial therapy acting on adhering stationary-phase microorganisms. In chronic osteomyelitis, orthopedic and plastic-reconstructive surgery is combined in the same procedure or within a short time span [9].

In our Wound Care Center, we see often that the patients refuse amputation even in tiny limbs like toes. They consider this to be mutilation and as an impairment of their quality of life. Owing to our low amputation rate or only internal amputation most patients come to the Wound Care Center to get a second opinion.

It would be ideal if after radical, surgical debridement of the bone with a local antibiotic could be inserted so that the success of antibiotic therapy does not depend entirely on orally taken antibiotics. Apart from this, it is possible that the remaining affected bone can be filled with substitute bone to stimulate build up of new bone.

\section{Methods}

We have developed the following six-point rules to treat an osteomyelitis ulcer:

(1) radical debridement;

(2) multisampling for bacteriology and histology;

(3) resection of all necrotic and infected bone;

(4) fill in an antibiotic-loaded resorbable bone-graft substitute in the residual bone;

(5) primary closure of the wound;

(6) avoid amputation if possible.

Between March 2015 and August 2015, a total of eight patients $(n=8)$ with clinical positive probe to bone test or radiological signs of osteomyelitis/osteitis treated in the wound center were included in this study.

Seven patients $(n=7)$ had an osteomyelitis and an ulcer, which was covered with fibrin. One patient $(n=1)$ had a wound, which rankled.

Excluded were patients with critical ischemia, which could not be resolved, or patients who suffered under serious general deterioration, which could not be stabilized.

All patients $(n=8)$ received a vascular routine checkup, an X-ray at two levels and a laboratory examination with infection parameters and glycemia.

In diabetic patients, the glycemia value was adjusted as necessary.

The 6-point plan was applied. The diseased part of the bone was resected, a radical debridement was done and a biopsy for bacteriology and histology of at least

Table 1 Analysis of bone and tissue.

\begin{tabular}{lll}
\hline Biopsy & Bacteriology & Histology \\
\hline Distal of infected bone & 1 & 1 \\
Infected bone & 1 & 1 \\
Prossimal of infected bone & 1 & 1 \\
\hline \multirow{2}{*}{ Tissue } & $\begin{array}{l}3 \text { (superficially, } \\
\text { intermittently, deep) }\end{array}$ \\
\hline
\end{tabular}


10 samples were taken (Table 1 ).

The debridement of the wound was done using jet lavage with one liter of $\mathrm{NaCl}$ and $500 \mathrm{~mL}$ solution of polyhexanid. Because it is so practical, the ultrasonic-assisted wound debridement was used when possible.

The residual bone was then drilled out and filled with antibiotic-loaded (gentamicin) resorbable bone-graft substitute under imager control (Fig. 1).

This procedure was performed on eight bones. In our last case, the antibiotic-loaded resorbable bone-graft substitute was not available. We filled the bone instead of a gentamicin sponge (Table 2).

For all patients $(n=8)$, a gentamicin sponge was placed in the wound cavity. If possible, the wound is primarily closed. In seven patients $(n=7)$, the wound was sutured immediately. In the two patients $(n=2)$, with malum perforans the ulcer was left open but access to the bone resection was closed.

In one patient $(n=1)$, the wound was initially left open because toes III and IV had to be amputated. Four weeks after surgery a secondary suture was made.

After surgery, a flat humid dressing was placed daily. In osteomyelitis of foot bones, a therapy-shoe was finally given to improve wound healing. After performing an antibiogram, intravenous or oral antibiotic therapy was prescribed by an infectiologist for a long term to avoid relapse.

After surgery, the wound was first checked daily, then weekly and after four weeks every two weeks, after six weeks for monthly and after three months every three months.

With the outpatient team, we were using telemedicine and, therefore, we were always available with e-mail or telephone (Fig. 2).

\section{Results}

Overall, of the eight patients $(n=8)$ (Table 3) included in the study we found nine bones with osteomyelitis/osteitis, positive bacteriological biopsies or positive radiological images. For all patients $(n=8)$ the probe to bone test was positive (Table 4).

Three patients $(n=3)$ had osteomyelitis of the metatarsal head I. One patient $(n=1)$ had osteomyelitis of the metatarsal heads III and IV and two $(n=2)$ patients had osteomyelitis of the metatarsal head $V$. Three toes had to be amputated because of humid necrosis. One patient $(n=1)$ had osteomyelitis of the

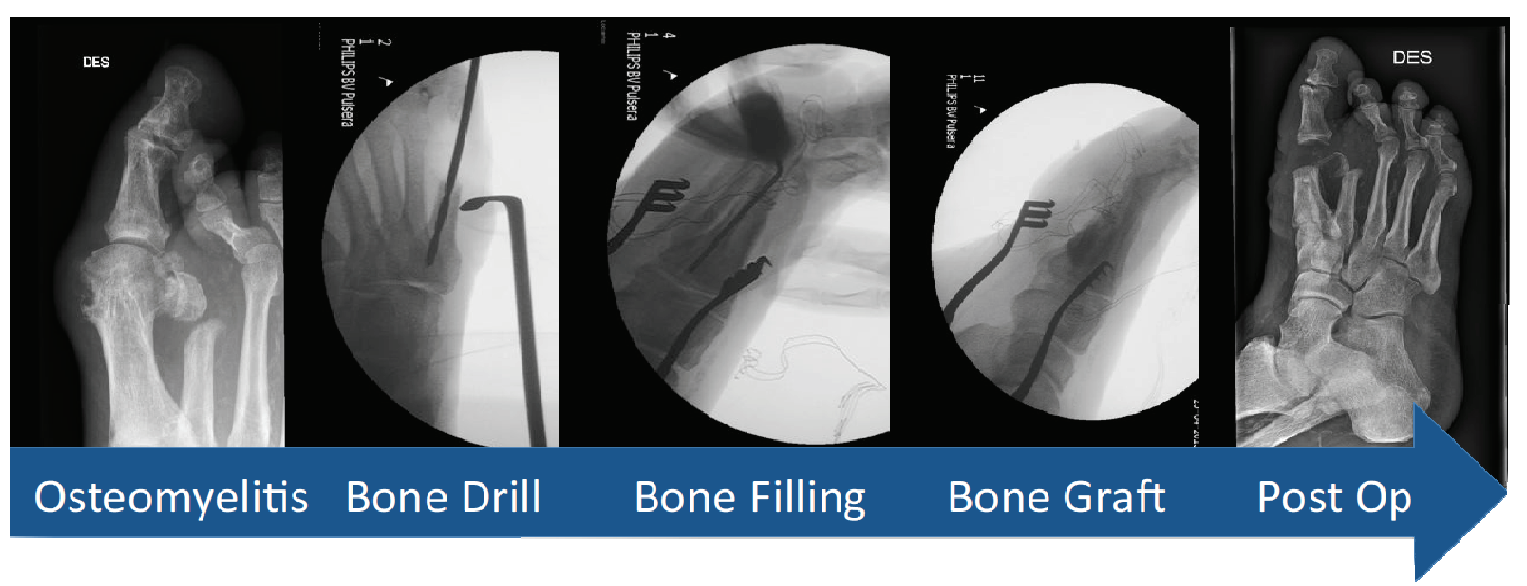

Fig. 1 Radiografic follow up.

Table 2 Surgery.

\begin{tabular}{lll}
\hline Items & & Values \\
\hline Bone filling & Antibiotic-loaded reabsorbable bone-graft Substitute & 8 \\
& Antibiotic-loaded reabsorbable sponge & 1 \\
\hline Wound cavity filling & Antibiotic-loaded reabsorbable sponge & 9 \\
\hline Amputation & A toe & 3 \\
\hline
\end{tabular}




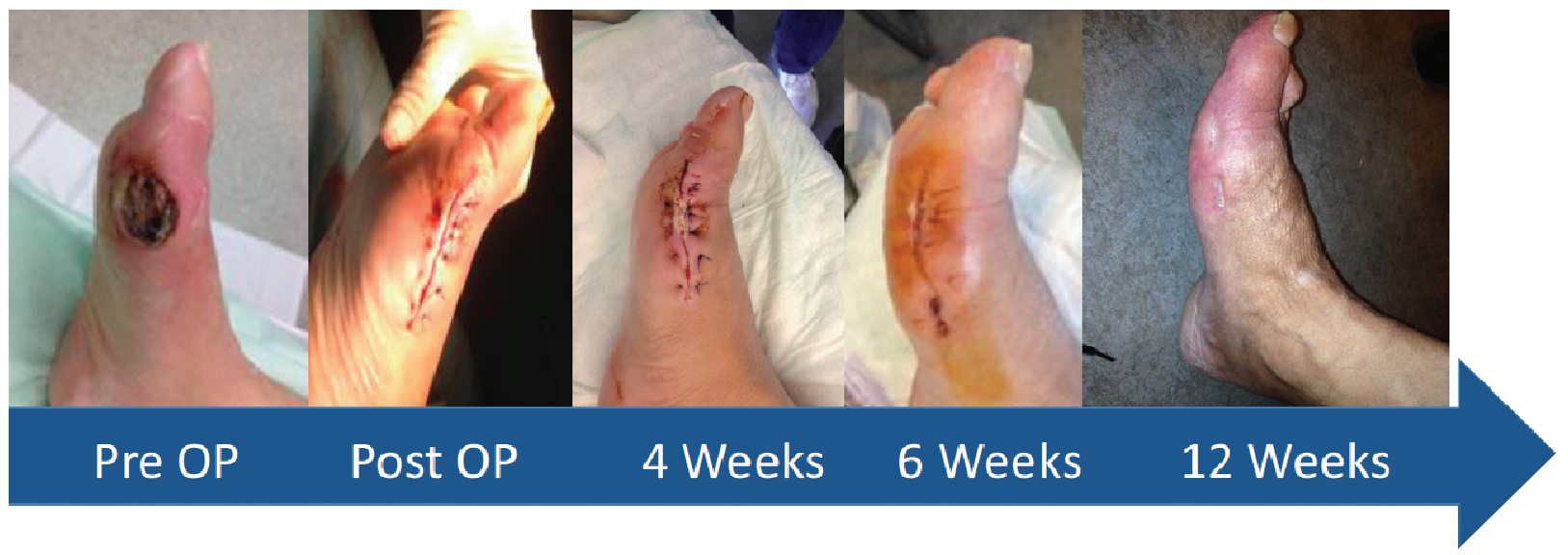

Fig. 2 Clinical follow up.

Table 3 Patient population.

\begin{tabular}{ll}
\hline Items & Values \\
\hline Patients & 8 \\
Men & 7 \\
Women & 2 \\
Mean age & 67 years \\
\hline
\end{tabular}

Table 4 Bone.

\begin{tabular}{ll}
\hline Items & Values \\
\hline $\begin{array}{l}\text { Infected bone (macroscopically, } \\
\text { bacteriologically, histologically or } \\
\text { radiologically) }\end{array}$ & 9 \\
\hline Probe to bone test & 9 \\
\hline Metarsal bone & $8(3 \times \mathrm{I}, 1 \times \mathrm{III}, 1 \times \mathrm{IV}, 3 \times \mathrm{V})$ \\
\hline Humerus & 1 \\
\hline
\end{tabular}

\section{Table 5 Concomitant disease.}

\begin{tabular}{ll}
\hline Items & Values \\
\hline Diabetes mellitus & 5 \\
Polyneuropathy & 6 \\
Polyneuropathy by diabetes mellitus & 5 \\
Polyneuropathy by chemotherapy & 1 \\
Peripheral arterial disease & 6 \\
Polyarthritis & 1 \\
Dialysis & 2 \\
Immunosuppression & 1 \\
Status after fracture and osteosynthesis & 1 \\
\hline
\end{tabular}

proximal humeral head (Table 4).

Six patients $(n=6)$ were male and two were $(n=2)$ female. The average age was 67 years (Table 3 ).

Five patients $(n=5)$ were suffering from diabetes mellitus and six $(n=6)$ from polyneuropathy. Six patients $(n=6)$ had been diagnosed with a peripheral arterial disease. One patient $(n=1)$ was in treatment with immunosuppression for polyarthritis. One patient $(n=1)$ was undergoing dialysis and one patient $(n=1)$ developed osteomyelitis after osteosynthesis of a fracture (Table 5).

The histology shows three osteomyelitis, three inflammations, one with additional gout and three without signs of osteomyelitis (Table 6).

The radiological examination shows two cases $(n=2)$ with signs of osteomyelitis and three $(n=3)$ without signs of osteomyelitis. In four $(n=4)$ patients, X-ray documentation was not available. The MRT examination shows two cases $(n=2)$ of osteomyelitis (Table 7).

Thirteen relevant germs were identified in the bacteriology. Twelve germs were sensitive to gentamicin and one was resistant (Table 8).

Table 6 Histology.

\begin{tabular}{ll}
\hline Items & Values \\
\hline Osteomyelitis & 3 \\
Osteoitis & 3 \\
Histology negative osteomyelitis & 3 \\
Osteomyelitis and gout & 1 \\
\hline
\end{tabular}

Table 7 Radiology.

\begin{tabular}{ll}
\hline Items & Values \\
\hline X-ray osteomyelitis & 2 \\
X-ray negative osteomyelitis & 3 \\
No X-ray picture available & 4 \\
MRT osteomyelitis primary/recurrence & $2 / 1$ \\
MRT negative for osteomyelitis & 1 \\
\hline
\end{tabular}


Table 8 Bacteriology.

\begin{tabular}{lll}
\hline Patient number & Bacterium & Gentamicin sensitive \\
\hline $\begin{array}{l}\text { Nr. 1 (Metarsal III) } \\
\text { Nr. } \text { (Metarsal IV) } \\
\text { (Patient Nr. 1 = Patient Nr. 2) }\end{array}$ & Citrobacter koseri & Yes \\
\hline Nr. 3 & & Yes \\
\hline Nr. 4 & Staphylococcus aureus & Yes \\
\hline Nr. 5a & Serratia marcescens & Yes \\
\hline Nr. 5b (relapse) & Staphylococcus epidermidis & No \\
\hline Nr. 6 & Klebsiella oxytoca & Yes \\
\hline Nr. 7 & Citrobacter freundii & Yes \\
\hline Nr. 8 & Pseudomonas aeruginosa & Yes \\
\hline Nr. 9 & Staphylococcus epidermidis & Yes \\
\hline Number of germs & Staphylococcus aureus & Yes \\
Number of germs sensitive to gentamicin & Staphylococcus epidemidis & Yes \\
Germ resistant to gentamicin & Staphylococcus epidermidis & Yes \\
\hline
\end{tabular}

Table 9 Antibiotic therapy.

\begin{tabular}{ll}
\hline Patient Number & Antibiotic therapy \\
\hline Nr. 1 (Metarsal III) & Tazobac, Rocefine \\
Nr. 2 (Metarsal IV) & \\
(Patient Nr. 1 = Patient Nr. 2) & Ciproxin, Rimactan \\
Nr. 3 & Antibiotic therapy after closure of the ulcer \\
Nr. 4 & Ciproxin \\
Nr. 5 a & Ciproxin \\
Nr. 5 b (relapse) & Zinaceff \\
Nr. 6 & Tavanic, Dalacin \\
Nr. 7 & Clindamycin \\
Nr. 8 & Bactrim forte \\
Nr. 9 &
\end{tabular}

Seven patients $(n=7)$ were treated orally with antibiotics based on the antibiogram. In one patient $(n=1)$ with malum perforans even received antibiotics after closure of the ulcer (Table 9). Only one patient ( $n=1)$ completed the three-month antibiotic therapy.

According to the classification of Cierny-Mader Stage there were six wounds with stadium III and three with stadium IV. In the classification Wagner Armstrong, there were three wounds with stadium IIIB and three with stadium IVB (Table 10).

For at least one week all patients $(n=8)$ had a discharge of serous fluid. In six patients $(n=6)$, seven bones healed immediately without problems. From this group, the dialysis patient $(n=1)$ died of a heart attack after 61 days follow-up. In two patients $(n=2)$ the suture had to be opened, due to excess antibiotic-loaded resorbable bone-graft substitute that was secreting from the suture.

In this case a phase-specific open wound treatment was conducted and both patients $(n=2)$ healed more than $80 \%$ but one ( $n=1$ ) died of a heart attack (Table 11 ).

Only one patient $(n=1)$ suffering from Morbus Buerger had a relapse osteomyelitis after cutting off his foot while swimming in the sea. Following another biopsy of the remaining metarsal V bone, an Ilomedinand antibiotic therapy was performed for three months with a healing rate of over $90 \%$. Actually, this patient does walk training. 
Table 10 Classification.

\begin{tabular}{lll}
\hline Items & Stadium & Values \\
\hline \multirow{2}{*}{ Cierny-Mader Stage } & III & 6 \\
& IV & 3 \\
\hline \multirow{2}{*}{ Wagner Armstrong } & 0 & 3 \\
& 3B & 3 \\
\hline
\end{tabular}

Table 11 Wound.

\begin{tabular}{ll}
\hline Items & Values \\
\hline Wound healed & 7 \\
\hline $\begin{array}{l}\text { Suture insufficiency (Patients Nr. 3 + 4) } \\
\text { Opening of the wound, removal of excess antibiotic-loaded resorbable bone-graft }\end{array}$ & 2 \\
substitute and open wound treatment & 2 \\
\hline Malum perforans (not closed after surgery) (Patients Nr. 4 +8) & 9 \\
Wound: fluid leakage & 7 \\
Primary wound closure of access to ulcer & 2 \\
Secondary wound closure (Patients Nr. 1+2) (Patient Nr.1 = Patient Nr. 2) & \\
\hline
\end{tabular}

Table 12 Survival time.

\begin{tabular}{ll}
\hline Items & Values \\
\hline Mean follow up & 5.77 months (2 11 months) \\
No recurrence & 8 \\
Recurrence of infection during follow-up (Patient Nr. 5) & 1 \\
Deceased from a heart attack (Patients Nr. 1+4) & 2 \\
\hline
\end{tabular}

Table 13 Follow-up.

\begin{tabular}{lllll}
\hline Patient Nr. & Surgery & Last control & Number of days & Number of months \\
\hline Nr. 1 & 04.03 .15 & 04.05 .15 & 61 & 2.03 \\
Nr. 1 & $30.03 .15^{*}$ & 04.05 .15 & & *wound suture \\
Nr. 2 & 04.03 .15 & 04.05 .15 & 61 & 2.03 \\
Nr. 2 & $30.03 .15^{*}$ & 04.05 .15 & & *wound suture \\
Nr. 3 & 30.07 .15 & 01.02 .16 & 186 & 6.2 \\
Nr. 4 & 03.09 .15 & 29.12 .15 & 117 & 3.9 \\
Nr. 5a & 11.03 .15 & 01.09 .15 & 174 & 5.8 \\
Nr. 5b & $02.09 .15^{*}$ & 01.02 .16 & 0 & *relapse osteomyelitis \\
Nr. 6 & 06.03 .15 & 01.02 .16 & 332 & 11.06 \\
Nr. 7 & 16.06 .15 & 01.02 .16 & 230 & 7.66 \\
Nr. 8 & 09.10 .15 & 01.02 .16 & 115 & 3.83 \\
Nr. 9 & 23.04 .15 & 01.02 .16 & 284 & 9.46 \\
\hline Total & - & - & 173.33 & 5.77 \\
\hline
\end{tabular}

Patient Nr. 1 = Patient Nr. 2;

Patient Nr. 1: Osteomyelitis metarsal III;

Patient Nr. 2: Osteomyelitis metarsal IV;

Patient Nr. 5b: Recurrent osteomyelitis, bone biopsy, debridement, Ilomedin and antibiotic therapy. On the radiograph image antibiotic loaded (gentamicin) resorbable bone-graft substitute is resorbed.

In total, seven patients ( $n=7)$ have survived without recurrent osteomyelitis with a mean follow-up of 5.77 months $(2 \sim 11$ months $)$. Two patients $(n=2)$ had a heart attack and died (Table 13). We have a Kaplan Meier curve with $80 \%$ survival without recurrent osteomyelitis in eleven months (Fig. 3 and Table 14). 


\section{Kaplan Meier Survival Curve}

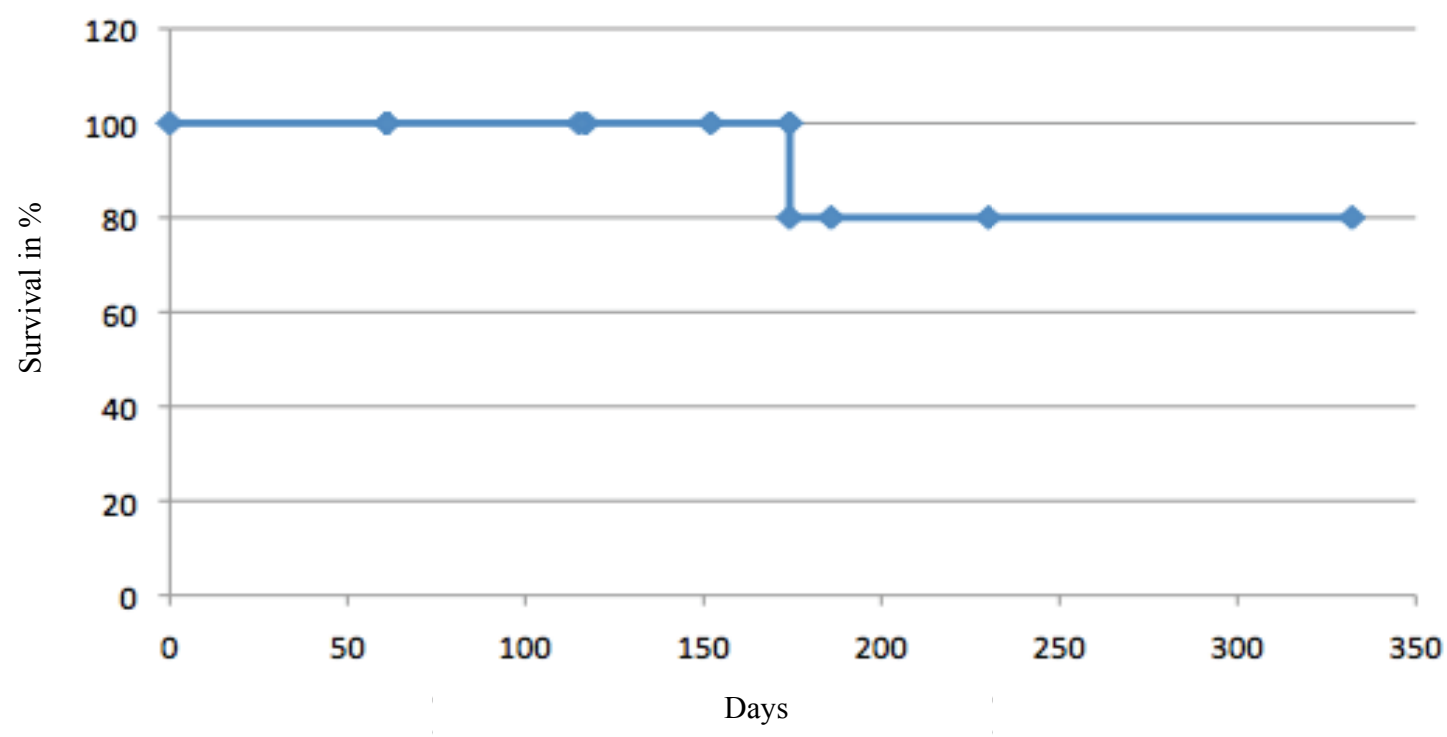

Fig. 3 Kaplan Meier curve.

Table 14 Kaplan Meier curve: survival.

\begin{tabular}{llllll}
\hline I: & Days: ti & The risk: ni & Event: di & $\begin{array}{l}\text { Number of survivors: } \\
\text { qi=(ni-di)/ni }\end{array}$ & $\begin{array}{l}\text { Cumulative survival rate: } \\
\mathrm{q} 1 * \mathrm{q} 2 * \ldots * \mathrm{q} i\end{array}$ \\
\hline Patient Nr. 1 & $61+$ & 9 & 0 & 1 & 1 \\
Patient Nr. 2 & $61+$ & 8 & 0 & 1 & 1 \\
Patient Nr. 8 & 115 & 7 & 0 & 1 & 1 \\
Patient Nr. 4 & $117+$ & 7 & 0 & 1 & 1 \\
Patient Nr. 5 & 174 & 5 & 1 & 0.8 & 0.8 \\
Patient Nr. 3 & 186 & 5 & 0 & 1 & 0.8 \\
Patient Nr. 7 & 230 & 5 & 0 & 1 & 0.8 \\
Patient Nr. 9 & 284 & 5 & 0 & 1 & 0.8 \\
Patient Nr. 6 & 332 & 5 & 0 & 1 & 0.8 \\
\hline
\end{tabular}

+ : censored, patients deceased.

\section{Discussion}

All patients $(n=8)$ had some serous fluid secretion but healed. We found that any excess bone-graft substitute must be completely removed; otherwise there will be prolonged secretion.

In three $(n=3)$ of the eight cases with metatarsal osteomyelitis, the toe had to be removed due to liquid necrosis. In five patients $(n=5)$, the toe could have been saved because there were no signs of infection. We did only an internal amputation. The patients themselves were very positive about the toe conserving therapy. For the patient a prevented amputation is a major positive point in their quality of life. Although pure anatomical and physiological stability and functionality of the toe is clearly different, none of the patients have felt the functional limitation as disturbing. For patients the toe-conserving therapy equals respect for their integrity. Psychologically this is a major advantage. Patients are aware that after the first amputation another can be expected within the following years. 
Each surgery was performed in one sitting. Another possibility is to perform a bone biopsy in the first sitting and then, according to the antibiogram, to choose the adequate graft (gentamicin or vancomycin) in a second sitting. Currently, two different antibiotic-loaded resorbable bone-graft substitutes are available (gentamicin or vancomycin).

The X-ray examinations during follow-up show that the antibiotic-loaded resorbable bone-graft substitutes have dissolved and strong bone formed.

The antibiotic-loaded resorbable bone-graft substitute (gentamicin or vancomycin) is available in a $5 \mathrm{~mL}$ or $10 \mathrm{~mL}$ package. Surgery can be performed on an outpatient basis saving expensive hospital costs. The graft inhibits spreading of the osteomyelitis and acts as a barrier against further infection of the bone.

The reliability of the patient when taking oral antibiotics is not so decisive, as there already is a local antibiotic depot.

We recommend this therapy only in patients with non-critical ischemia in macroscopic osteomyelitis in an operating theater setting.

The antibiotic-loaded resorbable bone-graft substitute has a high antibiotic effect locally. We often see that patients do not complete the three-month antibiotic treatment because of side effects such as abdominal pain or nausea, or because of the patient's very low compliance. The antibiogram in bone biopsy was often sensitive to gentamicin (in 12 cases $(n=12))$.

A clarifying question in future studies would be whether oral antibiotic therapy with gentamicin is at all necessary for sensitivity germs of bone biopsy.

To better understand the effectiveness of the therapy, a larger number of patients must be studied in the future with longer observation periods.

We consider the relatively high success and low complication rates to be the result of the local antibiotic depot inserted with the substitute bone after the surgical, radical debridement completes total eradication of the infection. The biggest advantage seems to be that after six months all the substitute bone is resorbed. This substitute bone has, however, built up solid new bone that shows up clearly on an $\mathrm{X}$-ray.

At the same time our six-point rule should be followed. Our 6-point plan turns out to be successful and we recommend it to other Wound Care Centers. Antibiotic-loaded resorbable bone-graft substitute is easy to use, has little wound complications and low recurrence rate.

\section{References}

[1] Kumar, V., Abbas, A. K., Nelson, F., and Mitchell, R. N. 2007. Robbins Basic Pathology 8th Edition. Philadelphia: Saunders Elsevier, 810-1.

[2] Mandell, G. L., Benett, J. E., and Dolin, R. 2005. Mandell, Benett and Dolin Principles and Practice of Infectious Diseases, 6th Edition. Philadelphia: Elsevier, 1322.

[3] Lew, D. P., and Waldvogel, F. A. 2004. "Osteomyelitis.” Lancet 364 (9431): 369-79.

[4] David, R., Barron, B. J., and Madewell, J. E. 1987. “Osteomyelitis, Acute and Chronic.” Radiologic Clinics of North America 25 (6): 1171-201.

[5] Lipsky, B. A., Berendt, A. R., Deery, H. G., Embil, J. M., Joseph, W.S., Karchmer, A.W., et al. 2004. "Diagnosis and Treatment of Diabetic Foot Infections.” Clinical Infectious Diseases 39: 885-910.

[6] Grayson, M. L., Gibbons, G. W., Balogh, K., Lewin, E., and Karchmer, A. W. 1995. "Probing to Bone in Infected Pedal Ulcers. A Clinical sign of Underlying Osteomyelitis in Diabetic Patients.” JAMA 273 (9): 721-3.

[7] Pineda, C., Vargas, A., and Rodríguez, A. V. 2006. “Imaging of Osteomyelitis: Current Concepts.” Infectious Disease Clinics of North America 20 (4): 789-825.

[8] Mader, J. T., Ortiz, M., and Calhoun, J. H. 1996. "Update on the Diagnosis and Management of Osteomyelitis." Clinics in Podiatric Medicine and Surgery 13: 701-24.

[9] Mader, J. T., Shirtliff, M., and Calhoun, J. H. 1997. "Staging and Staging Application in Osteomyelitis." Clinical Infectious Diseases 25 (6): 1303-9.

[10] Trampuz, A., and Zimmerli, W. 2006. "Diagnosis and Treatment of Infections Associated with Fracture-Fixation Devices.” Injury 37 Suppl. 2: 59-66. 